\section{A closer look at melancholia: saccadic eye movements in melancholic and nonmelancholic depression}

\section{Gurvich', P Fitzgerald', N Georgiou-Karistianis², O White ${ }^{3}$}

'Alfred Psychiatry Research Centre; ${ }^{2}$ Monash University; and ${ }^{3}$ Royal Melbourne Neurosciences, Melbourne, Australia

Background: Major depressive disorder may be a heterogeneous disorder; yet, melancholic depression is the most consistently described subtype, regarded as qualitatively different to nonmelancholic depression in terms of cognitive and motor impairments. Eye movement studies in depression are infrequent and findings are inconclusive.

Methods: This study used a battery of saccadic (very fast) eye movements to explore reflexive saccades, as well as higher order cognitive aspects of saccades including inhibitory control and spatial working memory. Nineteen patients with major depressive disorder (9 melancholic, 10 nonmelancholic) and 15 healthy controls participated.

Results: Differences were showed between melancholic and nonmelancholic patients. Melancholia was associated with longer latencies, difficulty increasing peak velocities as target amplitudes increased and hypometric primary saccades during the predictable protocol. In contrast, the nonmelancholic depression group performed similarly to controls on most tasks, but saccadic peak velocity was increased for reflexive saccades at larger amplitudes.

Conclusions: The latency increases, reduced peak velocity and primary saccade hypometria with more severe melancholia may be explained by functional changes in the fronto-striatal-collicular networks, related to dopamine dysfunction. In contrast, the serotonergic system plays a greater role in nonmelancholic symptoms and this may underpin the observed increases in saccadic peak velocity. These findings provide neurophysiological support for functional differences between depression subgroups that are consistent with previous motor and cognitive findings.

\section{Cognitive remediation in first-episode and chronic schizophrenia}

\section{A Harris', P Rogers'2, G Moore ${ }^{3}$, D Toscano ${ }^{4}$, A Redoblado-Hodge ${ }^{5}$}

${ }^{1}$ The University of Sydney; ${ }^{2}$ PEIRS, Sydney West Area Health Service; ${ }^{3}$ Macquarie Hospital; "Brain Dynamics Centre; and ${ }^{5}$ Redbank House, Sydney West Area Health Service, Sydney, Australia
Background: This study assessed the effectiveness of computer-assisted cognitive remediation in the treatment of cognitive deficits in people with both recent onset schizophrenia and chronic schizophrenia.

Methods: A randomized wait-list control study was conducted over eight sites using Medalia's Neuropsychological Approach to Remediation (NEAR). All subjects were diagnosed with schizophrenia or schizoaffective disorder and were recruited from a range of community and in-patient facilities. Subjects were randomized between an immediate treatment and a waitlist group, the later being treated after 15 weeks. Subjects were assessed at baseline, after at least 20 sessions of NEAR and 15 weeks after the completion of treatment on measures of symptomatology, function and neurocognition.

Results: Minimal differences were observed between waitlist and immediate treatment groups at baseline. However subjects with chronic schizophrenia were rated significantly higher for positive and total symptoms on the Positive and Negative Syndrome Scale. After treatment, significant improvements were observed for attention, processing speed and a limited range of executive functions. Improvements were accompanied by an improvement in social and occupational functioning particularly for subjects with recent onset disease. There were few changes in levels of symptomatology, self-esteem or quality of life.

Conclusions: This study supports the effectiveness of computer-assisted cognitive remediation in both recent onset and chronic schizophrenia in treating the cognitive deficits of schizophrenia. This appeared to have a social and occupational impact for young people with recent onset disease.

\section{Atypical antipsychotic use in Australian patients: cross-sectional study of uptake and perceived benefit}

\author{
C Harvey', G Hawthorne², H Herrman³, C Graham4, \\ A Favilla ${ }^{4}$ \\ 'Psychosocial Research Centre, Department of Psychiatry, The University of \\ Melbourne; ${ }^{2}$ Department of Psychiatry, The University of Melbourne; ${ }^{3}$ School of \\ Population Health, The University of Melbourne; and ${ }^{4}$ Formerly in the \\ Department of Psychiatry, The University of Melbourne, Melbourne, Australia
}

Background: Atypical antipsychotics are not as widely used in Australian public mental health as expected from evidence of their efficacy and treatment guidelines recommendations. We assessed the reasons for this from the perspectives of patients, their carers and clinicians.

Methods: A random sample of people with a diagnosis of schizophrenia attending four public mental 
health clinics in Melbourne $(n=83)$, their carers $(n=60)$ and their clinicians $(n=66)$ completed a questionnaire on the effectiveness, acceptability and side-effects of current, previous and early antipsychotic medicine. Medicine use was determined from clinical records.

Results: Patients were predominantly single middleaged women. A significant shift over time toward atypical medicine use had occurred: $66 \%$ were currently taking atypicals; compared with early medicines, current medicines were three times more likely to be atypical (odds ratio: 2.95, 95\% confidence interval: 1.48-5.88). Major discrepancies were noted in reports of medicines used between patients, carers, clinicians and clinical notes. Doctors made $61 \%$ of all recommendations for changes in previous medicines. There were few significant differences in perceived effectiveness, satisfaction and side-effects when comparing types of medicine. Health-related quality of life was associated with reported side-effects, but not with current medicine type.

Conclusions: There is a mutual lack of information and understanding about antipsychotic medicines between patients, their carers and clinicians. Greater reinforcement of provisions and incentives for collaborative treatment planning may be beneficial. Further studies of the uptake of atypical antipsychotics and their benefits should be conducted in real-world settings.

\section{A comparison of the implementation of assertive community treatment in Melbourne and London}

\section{Harvey', H Killaspy ${ }^{2,3}$, S Martino ${ }^{4}$, S White, S Johnson ${ }^{2,3}$}

Psychosocial Research Centre, Department of Psychiatry, The University of Melbourne, Melbourne, Australia; ${ }^{2}$ University College London; ${ }^{3}$ Camden and Islington Mental Health and Social Care Trust, UK; ${ }^{4}$ Private Psychiatrist, Glen Iris, Melbourne; and '5t George's University, London, United Kingdom

Background: Differences in implementation of assertive community treatment (ACT) could explain variability in reported effectiveness.

Methods: The Pan London Assertive Outreach (PLAO) studies examined ACT implementation and effectiveness amongst 24 London teams (Wright et al. 2003; Billings et al. 2003; Priebe et al. 2003). The current study gathered data on team organization, staff and client characteristics from four Melbourne ACT teams using identical participant sampling and data collection methods to the PLAO studies (except client characteristics were collected from Melbourne team staff rather than case notes).
Results: Melbourne teams were significantly different from London cluster $\mathrm{C}$ teams so comparisons were with cluster A and B teams only. All Melbourne teams worked extended hours; they took greater responsibility for dealing with crises than the London teams. Three of the four Melbourne teams achieved a majority $(>70 \%)$ of client contacts in vivo compared with only one third of the London teams. There were no significant differences between Melbourne and London teams regarding staff satisfaction and burnout. Client sociodemographic characteristics were very similar. Three quarters of all clients in both countries were admitted in the preceding 2 years but half the bed days were used in Melbourne.

Conclusions: An important difference in the implementation of ACT between Melbourne and London could be home visiting, a postulated 'active component' of models of home-based treatment. Melbourne teams may be more proactive in admitting patients at an earlier stage of relapse.

\section{The impact of the introduction of an acute sedation practice guideline}

\section{K Hirth ${ }^{1}$, A de Castella², M Dooley ${ }^{3}$, Y Hollander ${ }^{2}$}

${ }^{1}$ Alfred Hospital; ${ }^{2}$ Alfred Psychiaty; and ${ }^{3}$ Bayside Health, Monash University, Melbourne, Australia

Method: A retrospective audit of patients admitted to the Alfred Psychiatry in-patient facility from 1 October 2004 to 31 January 2006. Patient demographics, in-patient length of stay and medication usage data were extracted from the patient's medical record. High-dependency unit (HDU) length of stay was also calculated. Information regarding Code Blues, Medical Emergency Team calls, Code Greys, staff and patient incidents, and patient seclusion were also collected.

Results: There were a total of 1563 admissions to Alfred Psychiatry in-patient units during the total study period. Of these admissions, 614 included treatment in the HDU, and 313 of these HDU admissions were available for collection. Staff injuries decreased significantly from 18 in the preimplementation period to 6 in the postimplementation period $(P=0.02)$, while patient falls also decreased significantly from 17 to $1(P=0.0003)$, and patient-on-patient assaults decreased significantly from nine to zero $(P=0.007)$. The number and length of seclusions trended up but not significantly $(P=0.07)$. The use of midazolam trended down, which was clinically, but not statistically, significant. There was also a significant increase in the mean total dose of oral olanzapine used as a when-required medication from 7.2 to $22.2 \mathrm{mg}$ $(P=0.003)$. 